\title{
DIELECTRIC CHARGING EFFECTS ON PARYLENE ELECTROSTATIC ACTUATORS
}

\author{
Tze-Jung Yao, Ken Walsh, and Yu-Chong Tai \\ Electrical Engineering, 136-93, California Institute of Technology \\ Pasadena, CA 91125, USA, yao@mems.caltech.edu
}

\begin{abstract}
We report here the first characterization of dielectric charging effects on Parylene electrostatic actuators. High-resistivity Parylene in-between air gaps can cause undesirable charging effects due to air ionization when operating as electrostatic sensors/actuators at voltages as low as tens of volts. This undesirable effect can either lower the sensitivity of the sensor or increase the pull-in voltage of the actuator. It is further observed that Parylene actuators operating at high voltage could even show "bounce-back" and "pull-in voltage drift" problems. It is concluded that even for MEMS, attention must be paid to the operating voltages and the resistivity of the dielectrics.
\end{abstract}

Keywords: Parylene, electrostatic actuator, charging

\section{INTRODUCTION}

Room temperature CVD-deposited Parylene is a promising MEMS structural material due to its mechanical flexibility, excellent electric insulation and post-CMOS compatibility. Various Parylene MEMS devices have already been demonstrated such as thermopneumatic microvalves [1], rapid DNA separation/detection channels [2], shear stress sensors [3] and microspeakers [4]. Here, we are especially interested in making Parylene-based electrostatic actuators for microfluidic pumps and valves. Since Parylene is a good insulator, we expect that charging effects could be important in Parylene devices. Our study starts with making simple Parylene cantilever actuators. However, it is immediately observed that the fabricated actuators suffer many undesirable effects such as threshold voltage shift, bounce-back and actuation hysteresis. Although it is known $[5,6]$ that parasitic charges on dielectric can distort and/or shift device characteristics and functionalities, particularly in electrostatically actuated microstructures, this problem has not yet been thoroughly studied in MEMS devices. It is the purpose of this work to perform detailed study of various charging effects in Parylene electrostatic actuators and to develop a charge-balance model. Although the study is focused on Parylene, the model can be applied to other electrostatic MEMS devices.

\section{CANTILEVER DEVICES AND EXPERIMENTS}

The fabrication process of Parylene electrostatic cantilever actuators studied here is similar to [7] using photoresist as the sacrificial layer. Metalization is made by $10 \mathrm{~nm} / 100 \mathrm{~nm} \mathrm{Cr} / \mathrm{Au}$ evaporation for both the bottom and top electrodes. The finished cantilevers are $200 \mu \mathrm{m}$ wide, $2.5 \mu \mathrm{m}$ thick, but with lengths varying from $200 \mu \mathrm{m}$ to $1 \mathrm{~mm}$ as shown in Fig.1.

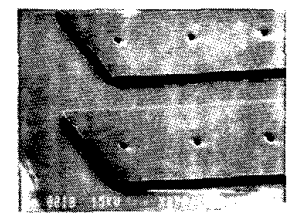

(a)

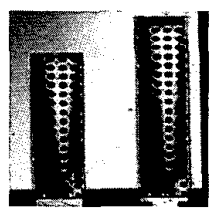

(b)

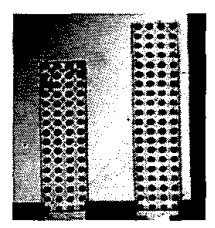

(c)
Fig. 1: Pictures of Parylene electrostatic cantilevers. (a) SEM picture (b) microphotograph of actuators at freestanding status (c) microphotograph of actuators at touch-down status

The ON (i.e. cantilever beam touches the substrate) and OFF states (i.e. cantilever beam is freestanding) can be clearly identified under microscope with the help of minute residual beam bending.

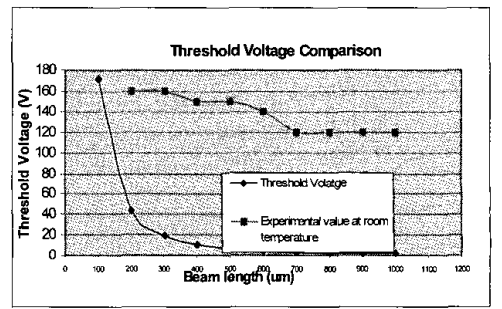

Fig. 2 Theoretical vs. experimental pull-in voltages of Parylene cantilever beams as a function of beam length

Electrostatic pull-in experiments are done on these actuators. Interestingly shown in Fig. 2, the pull-in voltages of fabricated electrostatic actuators show a very large discrepancy from their theoretical values [12]. Moreover, some of the devices even showed a "bounce-back" phenomenon with the continuously supplied bias voltage. Fig. 3 explains the experimentally observed "bounce-back" phenomenon, where the cantilever beam was first pulled down into contact with the substrate but it then bounced right back up into free-standing position when the pull-in voltage is continuously on. This "bounce-back" effect prevents the devices to operate properly and is the main focus of investigation here. We have then designed several experiments to further study the Parylene charging effects. First, we study the charge retention capability of Parylene using both conduction and ion deposited method. Then, the Parylene microstructures are bombarded with electrons and observed using SEM. Finally, based on these results we develop a quantitative model to explain the phenomena and provide some guidelines of choosing dielectrics and operating voltages for electrostatic actuators of the same kind. 


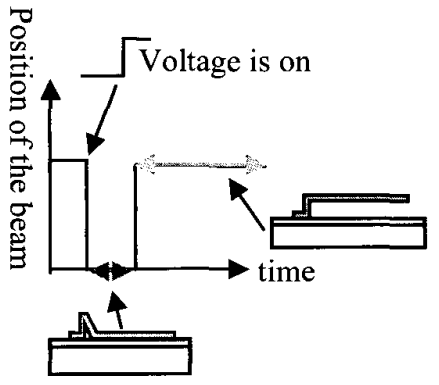

Fig. 3: Schematics of "bounce-back" effect.

\section{ELECTRICAL CONDUCTION IN PARYLENE}

Insulating materials such as Parylene have been used widely as electrical insulating materials. However, the high-field phenomena of insulating polymers are typically very complicated because of their chemical and physical structures and/or because of various additives or impurities in the polymer. In addition, space charges are known to affect electrical conduction and breakdown of the insulating material [8]. In this work, the dielectric strength of Parylene is investigated using parallel plate capacitors with different thickness of Parylene. The typical current-voltage characteristics are shownin Fig. 4. It is found that the electrical behavior of the Parylene thin-film is like a resistor with high resisitivity at low fields. However, the conduction current increases drastically beyond $2.5 \mathrm{MV} / \mathrm{cm}$ when breakdown begins. The high-field breakdown mechanism has been investigated by several groups and is believed to be caused by space charges inside Parylene under high electric field [8]. Therefore, it is possible that the charging effect of Parylene electrostatic actuators may be due to space charges inside the dielectrics. Interestingly as shown later, our study of vacuum tests has ruled out this possibility.

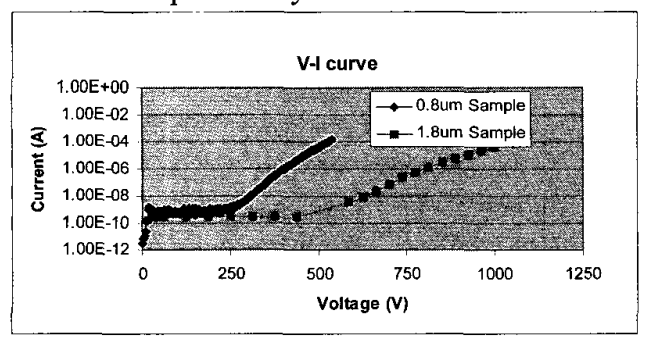

Fig. 4 Voltage-Current relationship of a Parylene dielectric capacitor prior to breakdown

\section{CHARGE RELAXATION MEASUREMENT IN PARYLENE}

Various methods of charging dielectrics have been developed in the past for electret applications such as triboelectric charging, isothermal charging, liquid contact charging, and ion beam charging. The charge retention capability of Parylene dielectrics is investigated here using ion beam charging with a Zerostat3 gun to create ionization of air molecules and inject the ions onto the dielectrics. As shown in Fig.5, Parylene is able to hold both positive and negative charges equally well with a measured charge relaxation time of approximately 4.64 hours.

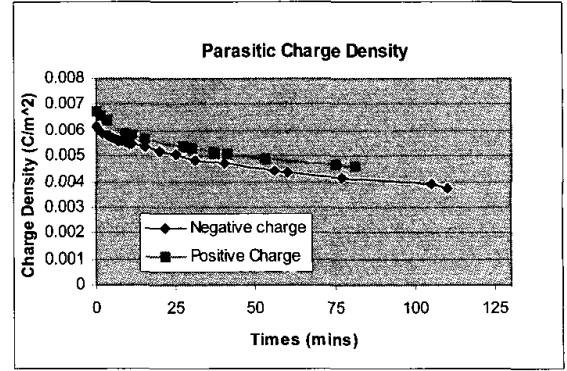

\section{Fig.5 Positive/Negative Parasitic charge accumulation on Parylene surfaces}

As in most electrets, the charge retention capability of Parylene thin film is decreased with elevated temperatures. Fig. 6 shows the effect of temperature to charge holding capacity of Parylene dielectrics. The measured relaxation time of Parylene thin-film at $120^{\circ} \mathrm{C}$ is less than 10 mins.

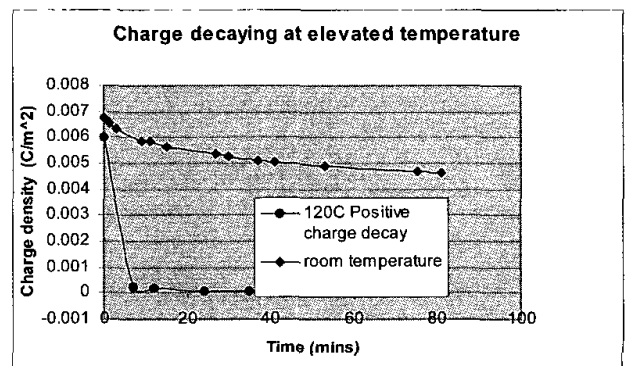

\section{Fig. 6: Positive charge decays at elevated temperatures $\left(120^{\circ} \mathrm{C}\right)$}

\section{CHARGING OF PARYLENE BEAMS UNDER SEM}

Earlier work on Analog Devices ADXL50 accelerometer under proton and radiation irradiation show that shifts in output voltages are caused by charging of dielectrics below the sensor [5]. It is well known that charging a dielectric is a contest between the emission of low-energy secondary electrons and the absorption of higher-energy electrons backscattered from other locations. It is also empirically known that dielectrics typically charge negatively when primary electrons hit the device at normal incidence at energies greater than $10 \mathrm{KeV}$ [9]. The Parylene microstructure in Fig.7 shows continuous upward movement under SEM observation. The details of fabrication of this device has been previously published [7]. This phenomenon is caused by charge accumulation at both the bottom of the cantilever beams and the top of the substrate. The accumulated negative charge thus provides a repulsive Columbic force to push the cantilever beam upward. 


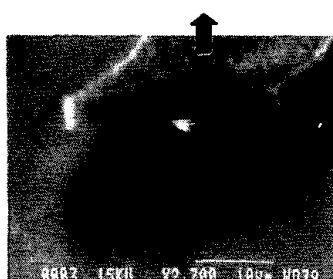

(a)

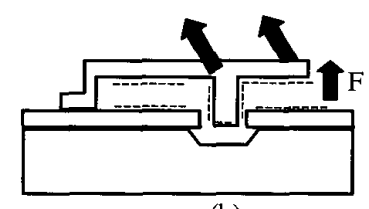

(b)
Fig.7 Movement of Freestanding cantilever beams under SEM

(a) SEM snaps of cantilever beams curving up

(b) Schematics of charge accumulation and the Columb force under SEM

\section{CHARGE-BALANCE MODEL}

Although it is known that the dielectrics in electrostatic sensors/actuators do get charged under either radiation[3] or high-field operation, the previous work [9] by Wibbeler showed that $\mathrm{SiO}_{2} / \mathrm{Si}_{\mathrm{x}} \mathrm{N}_{\mathrm{y}}$ stacks used as electrical insulation charged under electrostatic operations can influence characteristics of the devices.

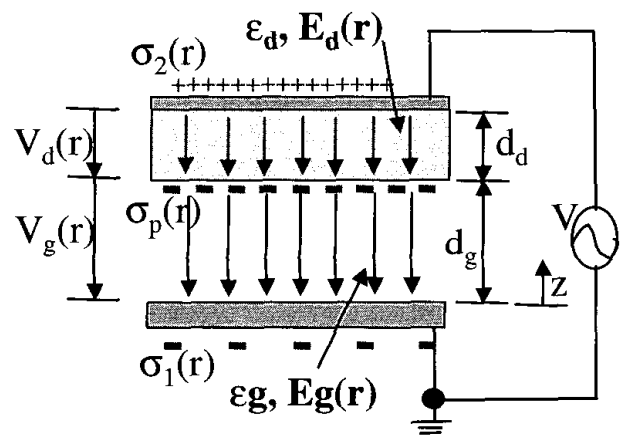

Fig. 8: Schematics of dielectrics charging process.

Considering an electrostatic actuator with interfacial charge $\sigma_{p}$ at the air-dielectrics interface shown in Fig.8, it has been proven [10] that the pull-in voltage of the cantilever beam will have to increase to compensate the field reduction in the air gap due to the interface charge. Therefore, the force and pull-in voltage of the cantilever system can be expressed as:

$$
\begin{aligned}
& F=\int_{(A)} d F=\frac{d C^{\prime}}{d z} \int_{(A)}^{\left[V-V_{p}(r)\right]^{2}} \frac{[}{2} d A \\
& \text { where } V_{p}=-\sigma_{p}(r) \frac{d_{d}}{\varepsilon_{d}} \text { and } \\
& V_{p u l l-i n}=V_{p}+\sqrt{\frac{18 E I d_{e f f}^{3}}{5 \varepsilon_{g} L^{4} w}} \\
& \text { where } d_{\text {eff }}=d_{g}+\varepsilon_{g} \frac{d_{d}}{\varepsilon_{d}}
\end{aligned}
$$

It is clear that the effect of the interface charge is to shift the pull-in voltage with its surface potential $\mathrm{V}_{\mathrm{p}}$. However the real situation is more complicated because the interface charge is a function of the air gap and the electric field applied on it. In order to predict the behavior of the cantilever beam under high electric field, it is necessary to understand the air ionization mechanism and the interface charge caused by air breakdown.

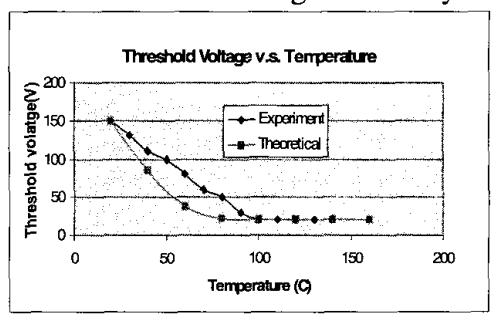

Fig.9 Threshold voltage as function of temperature. (Beam length $400 \mu \mathrm{m}$ ) vs. theoretical threshold voltage from chargebalance equation

Nevertheless, the interface charge model can explain why the pull-in voltages of Parylene cantilever beams (shown in Fig.2) significantly deviate from the theoretical charge-free value. It can be calculated that a $120 \mathrm{~V}$ pull-in voltage shift from theoretical values corresponds to an interface charge density of $1.34 * 10^{-3} \mathrm{C} / \mathrm{m}^{2}$ using Eq. 2 . The process to produce this interface charge can be categorized into three regions. First, at low field $(<3 \mathrm{MV} / \mathrm{m})$, the air medium is well accepted as good electrical insulator and thus the charge accumulation is negligible. However, at higher field $(\sim 3 \mathrm{MV} / \mathrm{m})$ the air medium starts to break down and the ionized charge particles start to travel across the air gap and deposit on the air/Parylene interfaces. Once the ions are deposited, the electric field within the air gap drops and brings the system back into the low field region. Detailed derivation shows that in this region (assuming the resistance of the ionized air medium is far smaller than the resistance of the dielectrics), the dielectrics would be charged up to the potential of applied voltage (i.e. $\mathrm{V}_{\mathrm{p}}=\mathrm{V}_{\text {appl }}$ ). When the voltage increases, the devices would enter the "saturation region," where current remains at a constant value over a large range of applied voltage. Therefore, the steady state interface charge can be derived as:

$$
\begin{aligned}
& \frac{\partial \sigma}{\partial t}=j_{0}-\frac{V_{p}}{r_{d} d_{d}}=0 \\
& \Rightarrow V_{p}=-j_{0} r_{d} d_{d}
\end{aligned}
$$

As Eq. 3 indicates, the pull-in voltage in this region is a function of Parylene thickness and resistivity. Due to high resitivity of Parylene dielectrics, Parylene electrostatic actuators suffer a high pull-in voltage shift. Moreover, the parameter $\mathrm{j}_{0=} 8 \times 10^{-8} \mathrm{~A} / \mathrm{m}^{2}$ calculated from Fig. 9 is known to be a function of electrode material and temperature. As Eq.3 predicts, the pull-in voltage of Parylene cantilever beams may also be a function of temperature. Shown in Fig.9, when the temperature of the cantilever increases, the resistance of the dielectrics drops and so does the trapped charges at the interface. As a result, the pull-in voltage of the cantilever drops and approaches the theoretical charge-free value. 


\section{DISCUSSION}

The more complex case is the "bounce-back" phenomenon, but this phenomenon can also be explained by air medium breakdown mechanism. When the cantilever moves closer to substrate, the electric field in the air gap increases and the air molecules continue to ionize. Although the breakdown of air is best described by the Paschen curve, which indicates high breakdown voltage of air medium in our case (minimum $\mathrm{V}_{\mathrm{br}}=340 \mathrm{~V}$ at $\mathrm{pd}=6-8 \mathrm{mbar}-\mathrm{mm}$ ), the avalanche secondary process may not be necessary to cause the "bounce-back" effect. Moreover, it has been reported by many groups $[10,11]$ that the Paschen curve is not valid when the air gap between electrodes is less than $4 \mu \mathrm{m}$, where the breakdown voltage at this range is significantly less than that predicted by the Paschen curve. For example, it is found that breakdown voltage can be as small as $20 \mathrm{~V}$ for a $1 \mu \mathrm{m}$ electrode gap. Therefore, the charges that cause the pull-in voltage shift may come from two sources: (1) the space-charge inside the Parylene film at high field (2) the breakdown of the air molecules. However, the "bounce-back" effect is not observed for our devices operated in high vacuum, where the pull-in voltage is closer to the theoretical value. This indicates that breakdown of the air medium is the main source of charges and that those charges can be eliminated by operating the device under vacuum. Furthermore, as shown in Fig.10a, the pull-in voltage of the cantilever beam will increase over the "bounce-back" cycles. This is caused by permanent charges deposited on the Parylene and has to be compensated again over the next cycles before the applied voltage can pull in the cantilever beams. The device under a higher electric field $(0.8-$ $1.2 \mathrm{MV} / \mathrm{cm}$ ) often displays the "snap-down" effect. The "bounceback" cycles deposit permanent charges on the Parylene beams, causing the cantilever beam to snap down to the substrate even when the applied voltage is removed. To bring the cantilever back to freestanding status, an external voltage is required to cancel the pull-in force generated by permanent charges as shown in Fig.10(b).

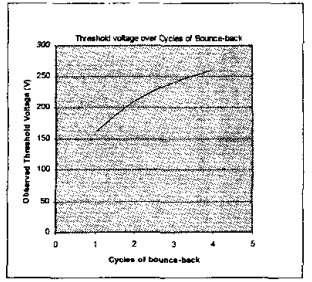

(a)

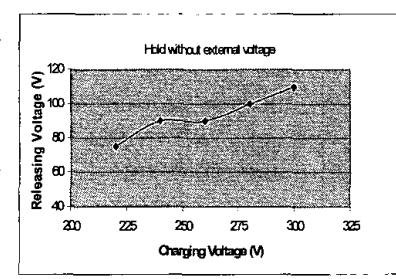

(b)
Fig. 10 Hysteresis Behavior of the Bounce-Back effect (a)The threshold voltage vs. the number of the bounceback cycles

(b) The hold-up voltage required to pull-up "snappeddown"cantilevers

\section{CONCLUSION}

This study characterizes the charging properties of Parylene dielectrics; the observed charging effects may also be applicable in other dielectrics. Charge accumulation and temperature dependence are explained by a charge-balance theory. In order to prevent the undesired "bounce-back" effect, the device should be designed to avoid electric fields higher than $150 \mathrm{~V}(0.56 \mathrm{MV} / \mathrm{cm})$, in particular those fields which are higher than $220 \mathrm{~V}(0.88 \mathrm{MV} / \mathrm{cm})$, in order to prevent permanent charge accumulation. Thus, for electrostatic actuators to be operated at high field $(>3 \mathrm{MV} / \mathrm{m})$, they should be designed in such a way as to avoid dielectrics between driving electrodes or to separate driving and sensing electrodes to prevent undesired parasitic charging effect.

\section{ACKNOWLEDGEMENT}

This work is supported by Agilent Technologies and the NSF Center for Neuromorphic System Engineering (CNSE) at Caltech.

\section{REFERENCES}

1. X. Yang, C. Grosjean, Y.C. Tai and C.M. Ho, A MEMS Thermopneumatic Silicone Rubber Membrane Valve, Sensors and Actuators A:Physical, Vol. 64, pp. 101-108, 1998.

2. J. Webster, Monolithic Structures for Integrated Capillary Electrophoresis Systems, Ph.D thesis, University of Michigan, 1999

3. X.Q. Wang, Z. Han, F. Jiang, T. Tsao, Q. Lin, Y.C. Tai, V. Koosh, R. Goodman, J. Law and C.M. Ho, A Fully Integrated Shear Stress Sensor, 1999 International Conference on Solid-State Sensors and Actuators (Transducers '99), Sendai, Japan, Jun.(1999).

4. C.-H. Han and E.S. Kim, Parylene-Diaphragm Piezoelectric Acoustic Transducers, IEEE International Micro Electro Mechanical Systems Conference, Miyazaki, Japan, January 23-27, 2000

5. Edmonds, L.D., G.M. Swift, and C.I. Lee, Radiation Response a MEMS Accelerometer: An Electrostatic Force. IEEE Transactions on Nuclear Science, 1998.45(6) pp.27792788.

6. W.C. Tang et al., J. Microelectromech. Syst. 1, pp.170-178 (1992)

7. T.J. Yao, X.Yang, and Y.C. Tai, $\mathrm{BrF}_{3}$ Dry Release Technologies for Large Freestanding Parylene MEMS, 2001 International Conference on Solid-State Sensors and Actuators (Transducers '01), Munich, Germany, Jun.(2001).

8. Mori, T., T. Matsuoka, and T. Mizutani, The Breakdown Mechanisms of poly-p-xylyene Film. IEEE Transactions on Dielectrics and Electrical Insulation, 1994.1(1)?p.71-76.

9. Jürgen Wibbeler, Günter Pfeifer, and M. Hietschold, Parasitic charging of dielectric surfaces in capacitive microelectromechanical systems (MEMS). Sensors and Actuators A, 1998.71 pp.74-81

10. Gemer, L.H., Electrical Breakdown between close Electrodes in Air. Journal of Applied Physics, 1959.30(1) pp.46-51.

11. Torres, J.-M. and R.S. Dhariwal, Electric field breakdown at micrometre separations in air and vaccum. Microsystem technologies, 1999.6 pp.6-10.

12. Petersen K.E., "Silicon as a Mechanical Material", Proc. IEEE, vol.70, no.5, pp.420-457, May 1982 